\title{
Interactive comment on "Lagged variation of moisture conditions in central Asia compared with monsoonal Asia during the last four interglacials" by Jia Jia et al.
}

\section{Anonymous Referee \#1}

Received and published: 6 December 2019

The study by Jia et al. deals with an interesting loess-palaeosoil section in Central Asia (Darai Kalon) with the concern to reconstruct palaeoenvironmental conditions for the last four glacial-interglacial cycles. Furthermore, the results are compared to a loess section from the Chinese Loess Plateau (Xifeng) in order to find spatial and temporal relations between humidity periods and atmospheric circulation patterns, especially related to the interaction between westerlies, the Indian Summer Monsoon and the East Asian Summer Monsoon.

In its present form, this study reveals some weak points that mainly relate to the methodological approach for establishing chronologies for the loess sections. 
(I) First, no independent age determination is presented that could support the chronology that is solely based on the assumed relation between grain sizes and $\mathrm{d} 180$ values from marine cores. Thus, the whole chronology remains very speculative. If the chronologies of loess sections from different areas are both established based on a peak matching between the grain-size curves and one d180-curve, of course the resulting curves are synchronous - that is circular reasoning.

(II) Second, the direct correlation between grain-size values / magnetic parameters and the Specmap-isotope curve implies that the built-up of the curves (in the sense of the built-up of the loess-palaeosoil sequences from bottom to top) is a continuous, successive and more or less linear process. But loess sedimentation rates are highly variable and may even tend towards zero during interglacial periods (see e.g. the study by Frechen and Dodonov (1998) at the same section, cited in the manuscript). That means that phases of lacking (or strongly reduced) loess deposition that relate to periods of pedogenesis (that may extend over several thousands of years) are ignored in the age model in favor of producing a kind of continuous "loess graph" assuming uniform deposition rates between the time control points. In my opinion, beside all other uncertainties of the chronology, already this linear interpolation between a few age control points is a strong contradiction and produces large errors. This is all the more serious if the interpretation strongly relies on assumed time lags in the range of a few thousand years. Furthermore, pedogenic clay formation that leads to finer grain sizes in subsoil horizons is a secondary process that is decoupled from primary aeolian deposition, but it strongly affects the mean grain-size curve. The approach to directly correlate the gradual fining of the grain-size in a loess depth profile with a linear progression of time (e.g. sedimentary d18O-curves, SPECMAP) is far away from the general concept of the way in which soils are formed. In my opinion, this also applies to magnetic susceptibility curves as soon as it is assumed that increasing values are caused by pedogenic processes instead of sedimentary processes. Such an approach could be just about acceptable for the aim to e.g. make a general charcterisation of soil formation or for realizing a rough comparison or correlation of different soils from

Interactive comment
Printer-friendly version

Discussion paper

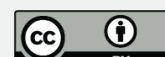


different sections, but not for generating a precise chronology for pedogenically altered loess sections.

(III) Third, as I understand it, the interpretation of time lags is strongly based on the respective position and the possible offset between peaks of the grain-size and frequency dependant susceptibility curves. Or alternatively, if a palaeosoil shows a thicker subsoil horizon and a less rapid clay decline with depths, also the frequency-dependant susceptibility may show a less rapid decline as these values are generally strongly correlated with clay contents. This may lead to a different or shifted slope of the susceptibility curve. In that case the assumed time lags are again caused by pedogenic processes, i.e. different soil development depths. Therefore, despite value curves/ peaks of different sedimentological/pedogenetic parameters appear in different soil depths, both may relate to the same formation time. Without showing the proxy-curves in a clear stratigraphic context, it is not possible to evaluate the plausibility of the used approach. Furthermore, it would be necessary to show the strength of correlation between clay and frequency-dependant susceptibility (FDS) values, because if they show a strong correlation, FDS is not an independent value and thus even more severely influenced by the tuning process.

Finally, considering these mentioned points that all concern the reliability of the age model, which in turn is the base for the interpretation, I cannot recommend the manuscript for publication in "Climate of the Past" in its present form.

Interactive comment on Clim. Past Discuss., https://doi.org/10.5194/cp-2019-117, 2019.

Interactive comment 